\title{
Electronically Modulated CoP by Ce Doping as a Highly Efficient Electrocatalyst for Water Splitting
}

Jiajie $\mathrm{Li}^{1}$, Shanbao Zou ${ }^{1}$, Xundao Liu ${ }^{1}$, Yue $\mathrm{Lu}^{1}$, Dehua Dong ${ }^{1, *}$

1. School of Materials Science and Engineering, University of Jinan, Jinan, 250022, P. R. China

* To whom correspondence should be addressed. E-mail: mse_dongdh@ujn.edu.cn; Tel: (+86) 531-89736011. Fax: (+86) 531-89736011.

Total number of pages: 12

Total number of figures: 13

Total number of tables: 4

\section{Table of Contents}

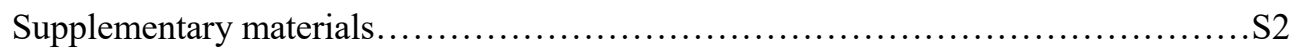

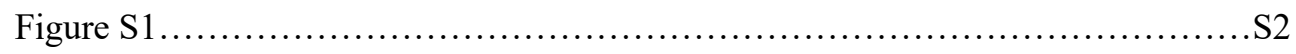

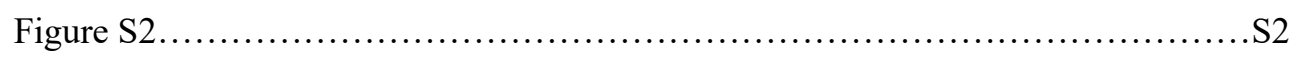

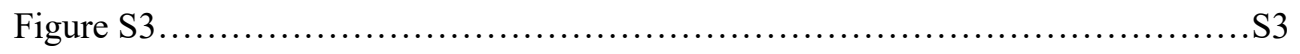

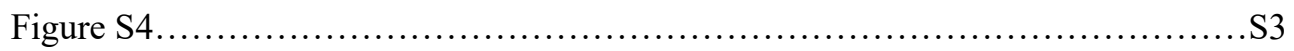

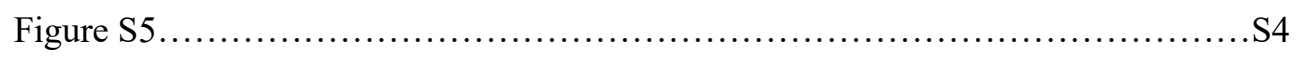

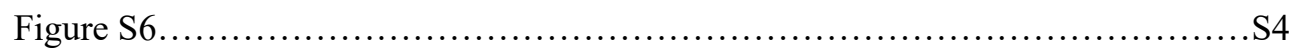

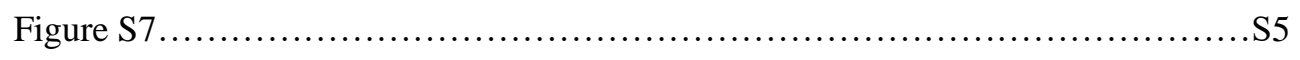

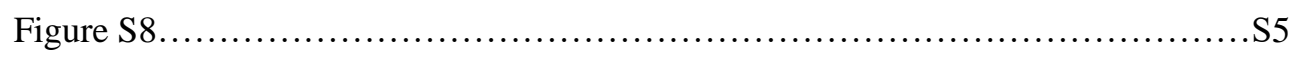

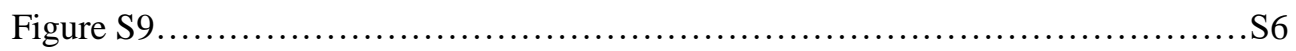

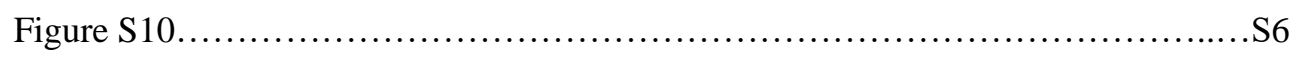

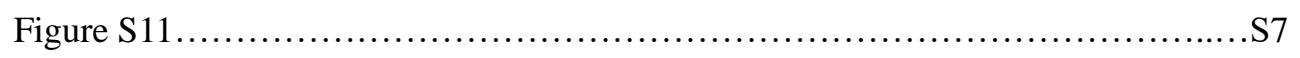

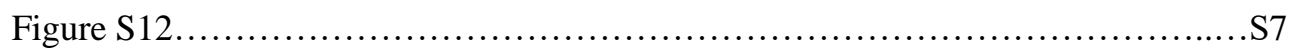

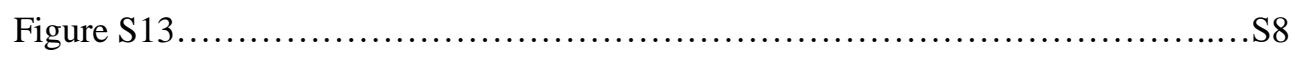

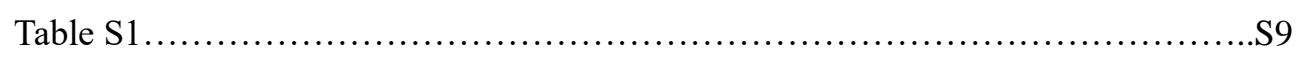

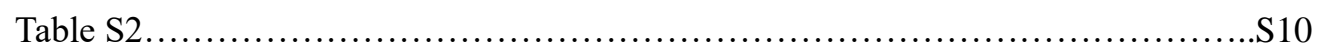

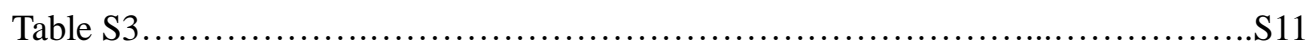

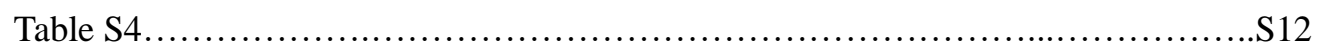



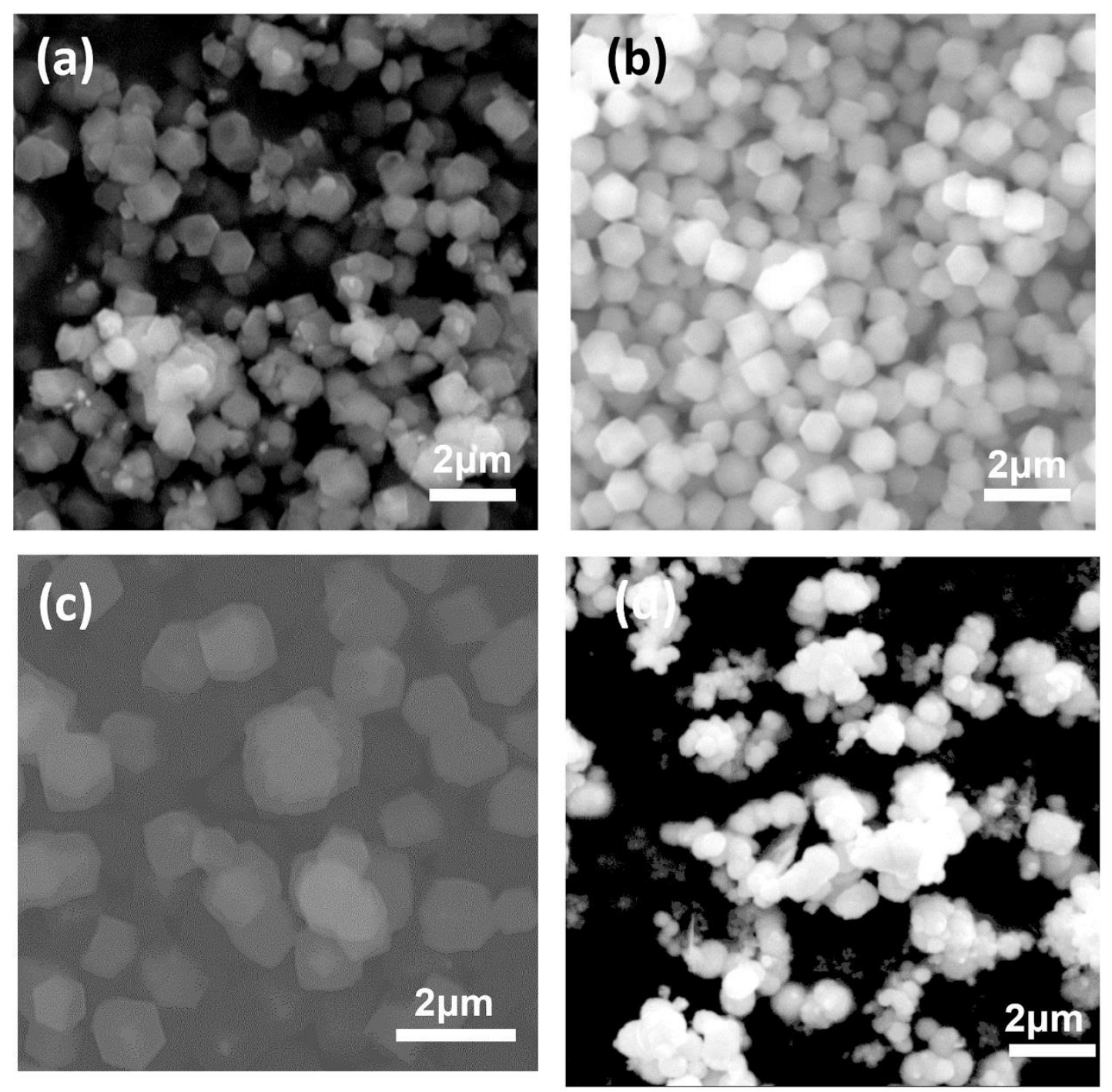

Figure S1 SEM image of (a) ZIF-67, (b) 0.5\%Ce-ZIF-67, (c) 2\%Ce-ZIF-67, (d) CeOx.
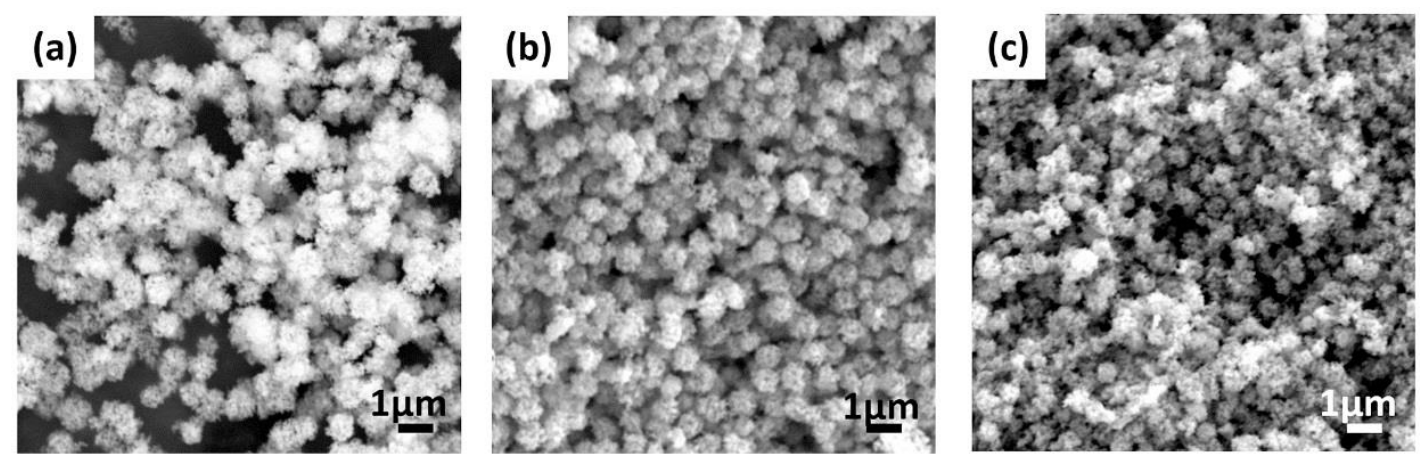

Figure S2 SEM image of the phosphorization product (a) $\mathrm{CoP}$, (b) $\mathrm{Ce}_{2}-\mathrm{CoP}$, (c) $\mathrm{Ce}_{0.5}$-CoP. 


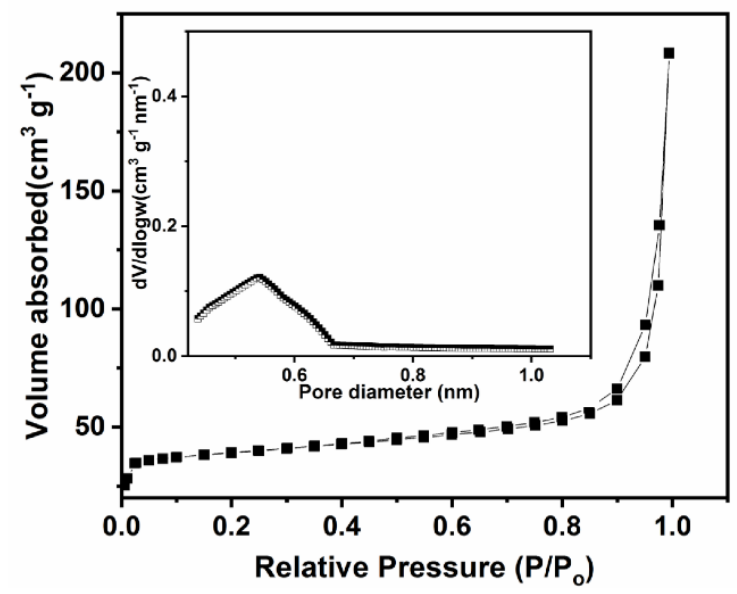

Figure $\mathrm{S} 3 \mathrm{~N}_{2}$ absorption/desorption isotherms and pore size distribution of $\mathrm{Ce}_{1}-\mathrm{CoP}$.

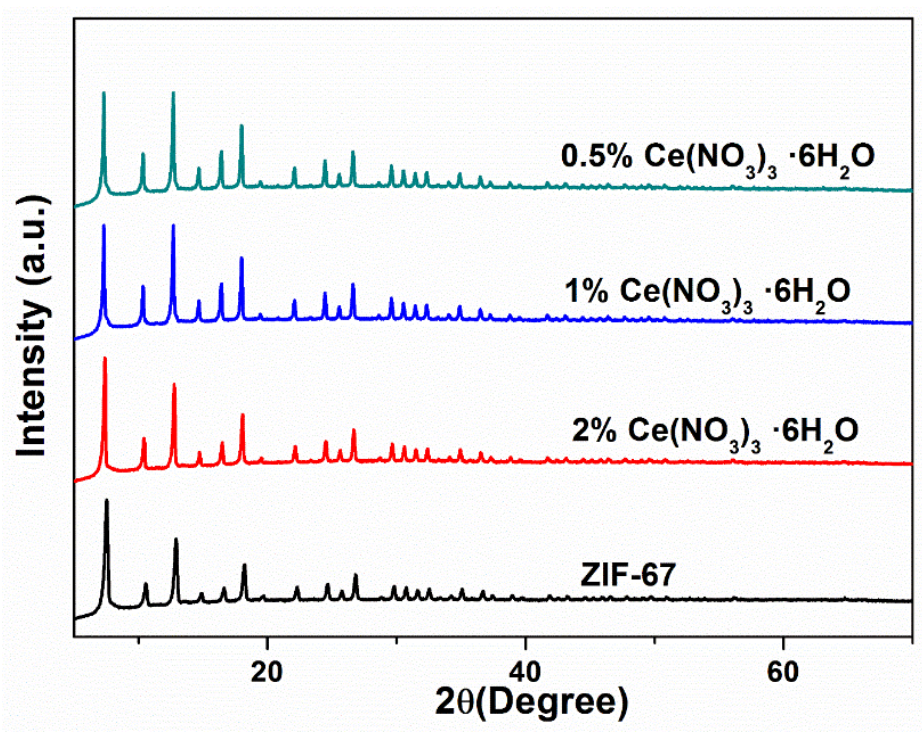

Figure S4 XRD patterns of Ce-ZIF-67. 


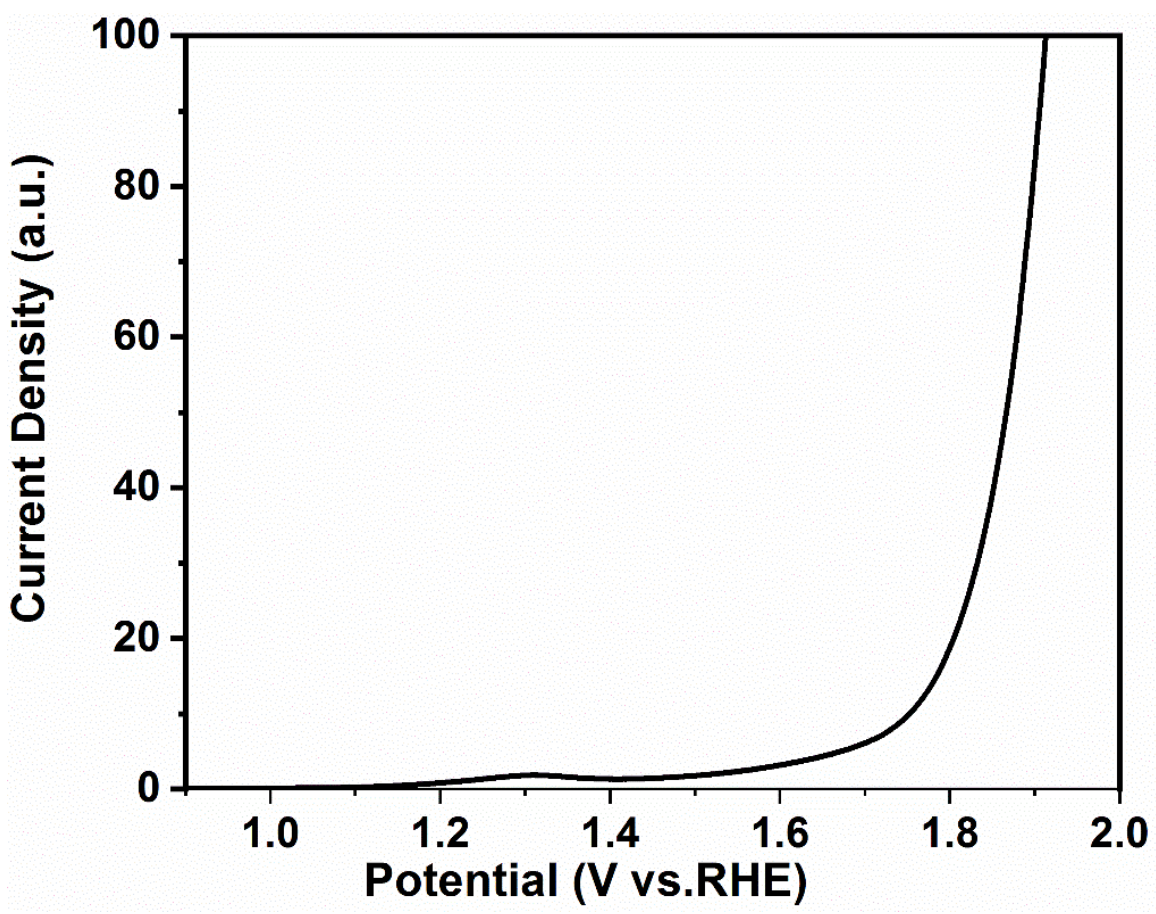

Figure S5 The pristine polarization curve of $\mathrm{Ce}_{1}-\mathrm{CoP}$ in $1 \mathrm{M} \mathrm{KOH}$ aqueous solution at $5 \mathrm{mV}^{-1}$ for OER.
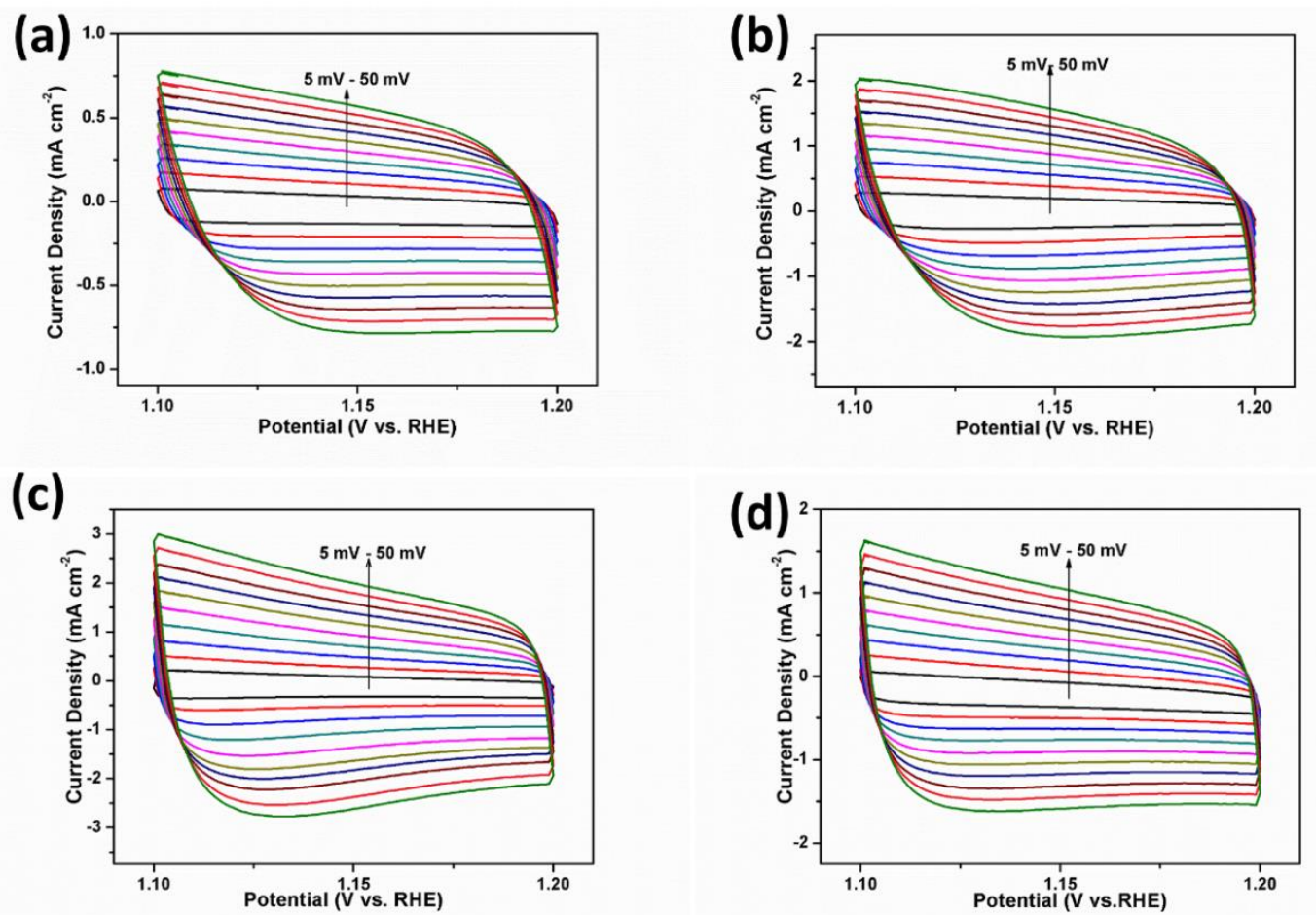

Figure S6 Cyclic voltammograms at the scan rate of $5 \mathrm{mV} \mathrm{s}^{-1}$ to $50 \mathrm{mV} \mathrm{s}^{-1}$ for (a) CoP, (b) $\mathrm{Ce}_{2}-\mathrm{CoP}$, (c) $\mathrm{Ce}_{1}-\mathrm{CoP}$, (d) $\mathrm{Ce}_{0.5}-\mathrm{CoP}$ in $1 \mathrm{M} \mathrm{KOH}$ solution in the region of 1.1-1.2 V vs. RHE. 


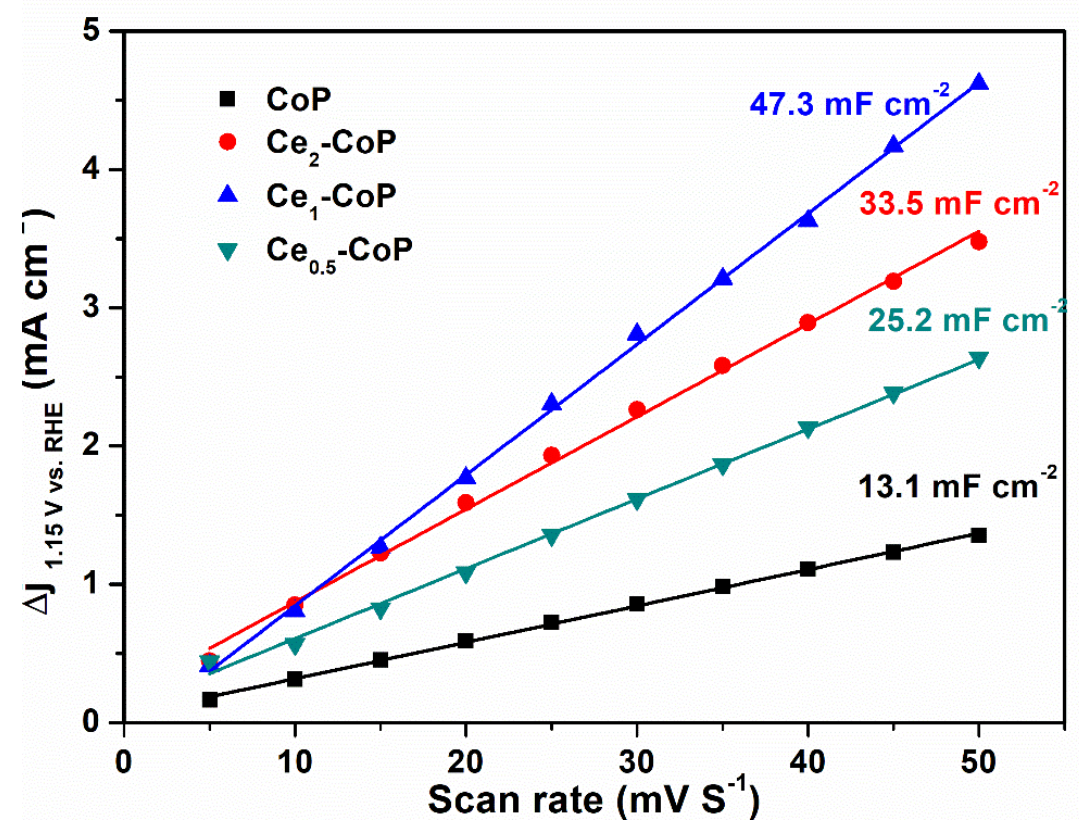

Figure $\mathrm{S} 7$ The calculated $\mathrm{C}_{\mathrm{dl}}$ for $\mathrm{CoP}, \mathrm{Ce}_{2}-\mathrm{CoP}, \mathrm{Ce}_{1}-\mathrm{CoP}$, and $\mathrm{Ce}_{0.5}-\mathrm{CoP}$.

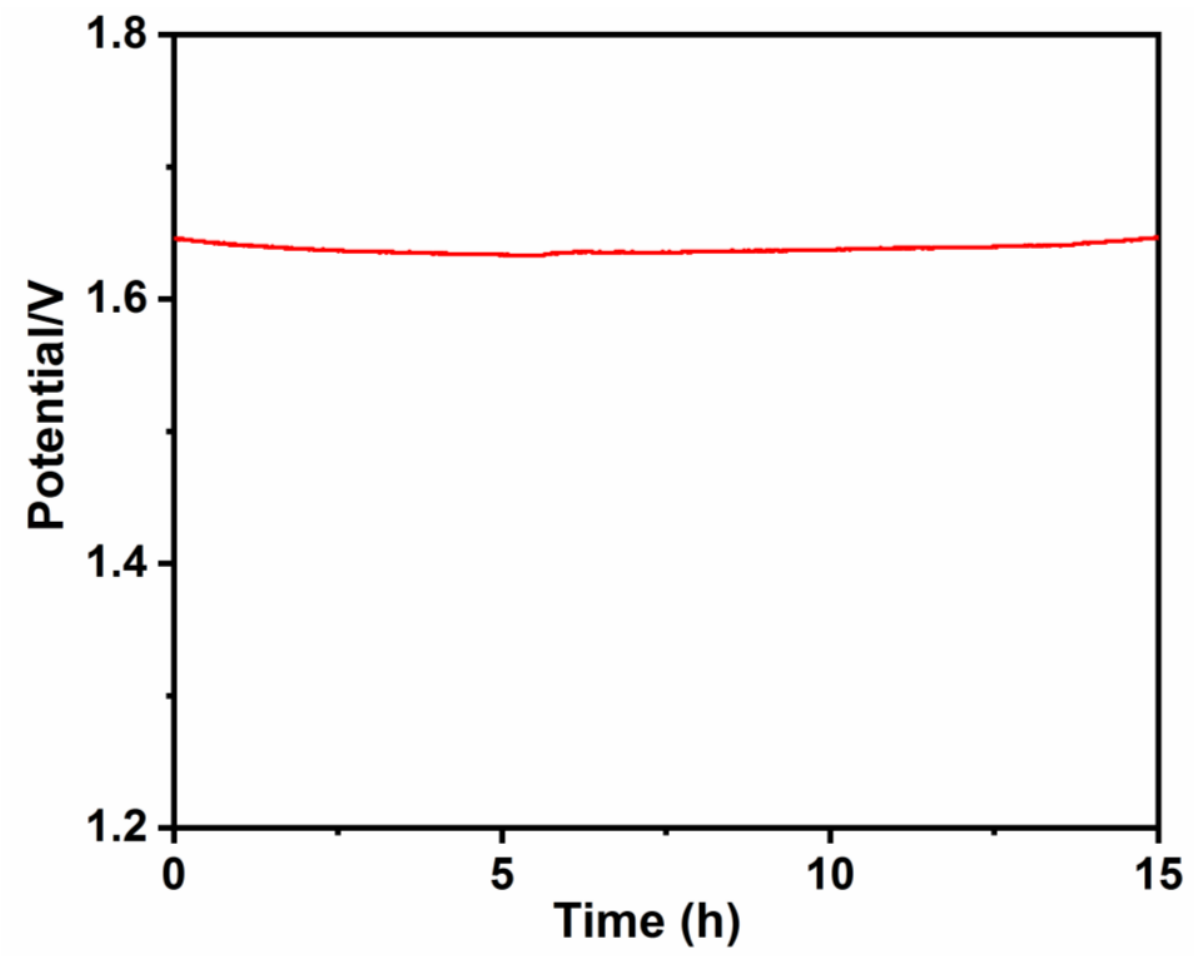

Figure S8 Chronopotentiometric curve of water electrolysis at a current density of 10 $\mathrm{mA} \mathrm{cm}{ }^{-2}$ 


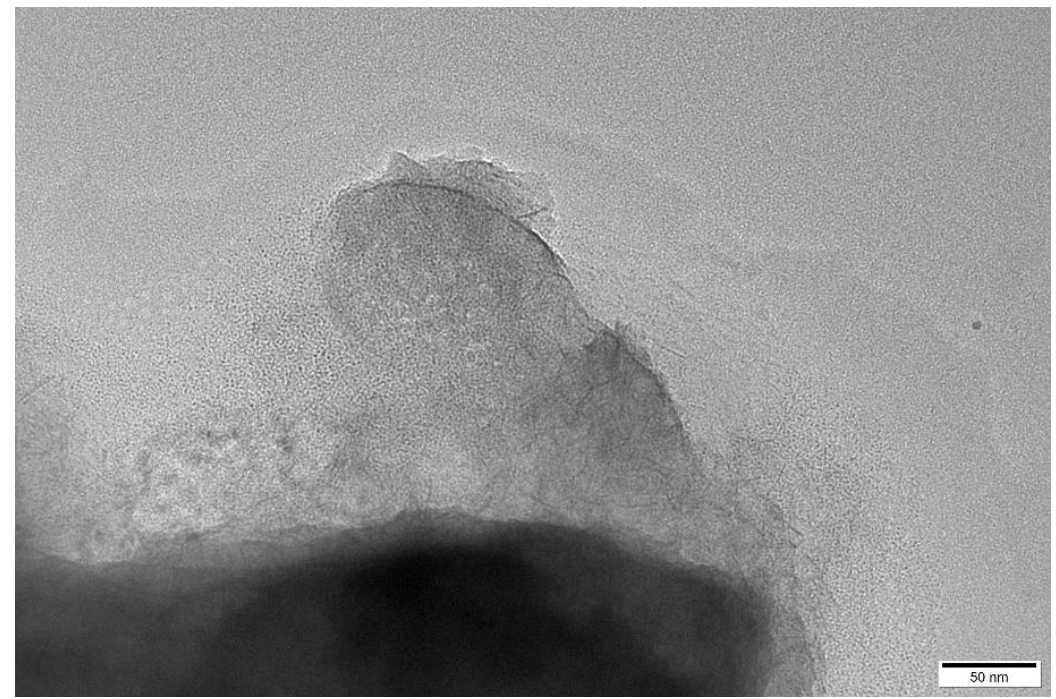

Figure S9 TEM image of the $\mathrm{Ce}_{1}-\mathrm{CoP}$ catalyst after long term test in $1 \mathrm{M} \mathrm{KOH}$ for OER.
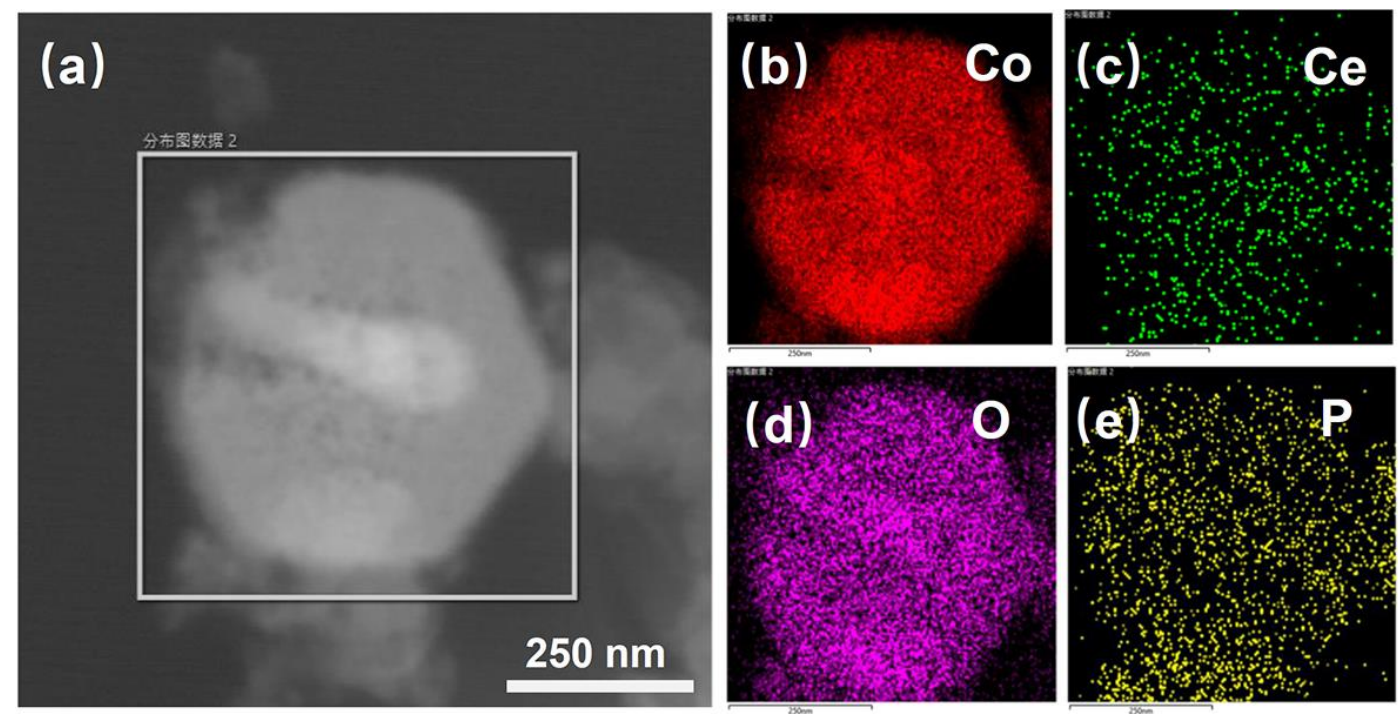

Figure S10 (a) STEM image and (b-e) the corresponding mapping image of $\mathrm{Ce}_{1}-\mathrm{CoP}$ after OER test. 

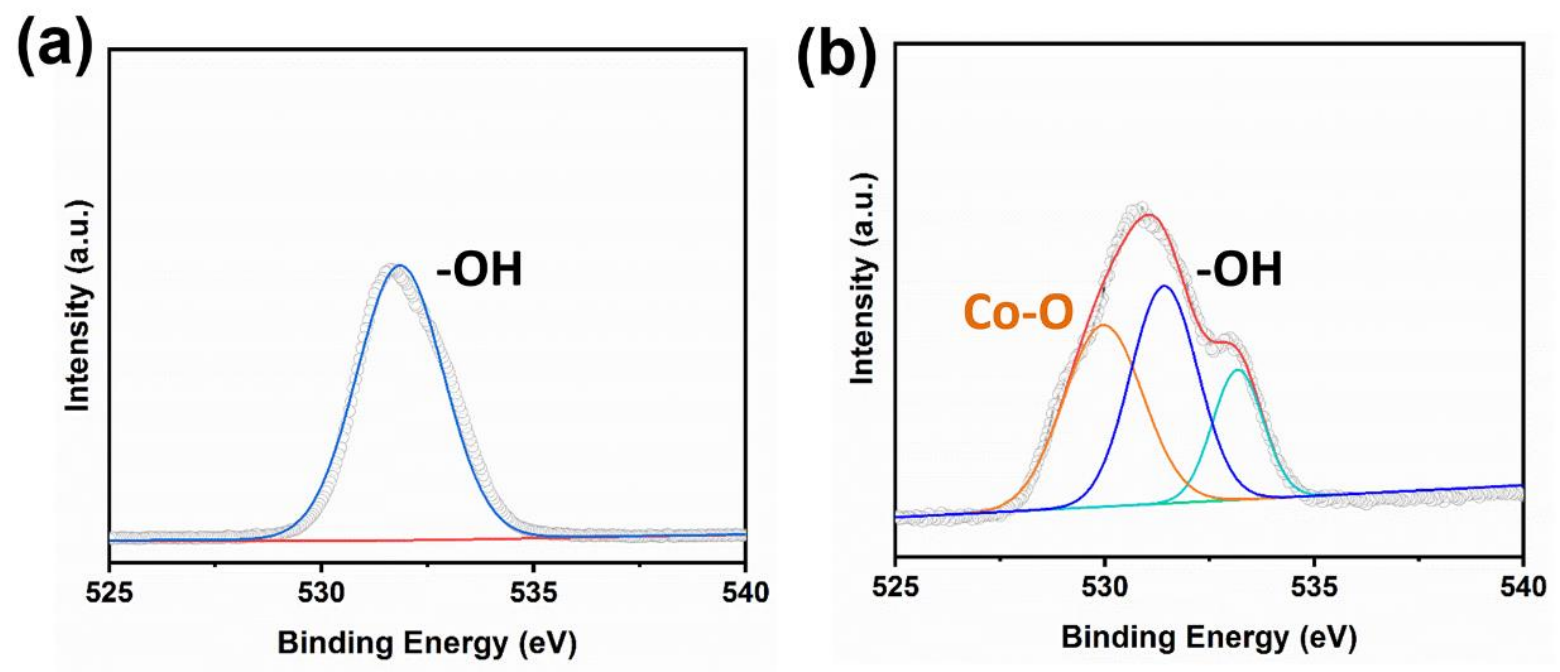

Figure S11 O1s XPS spectrum of $\mathrm{Ce}_{1}-\mathrm{CoP}$ (a) before and (b) after the OER stability tests.

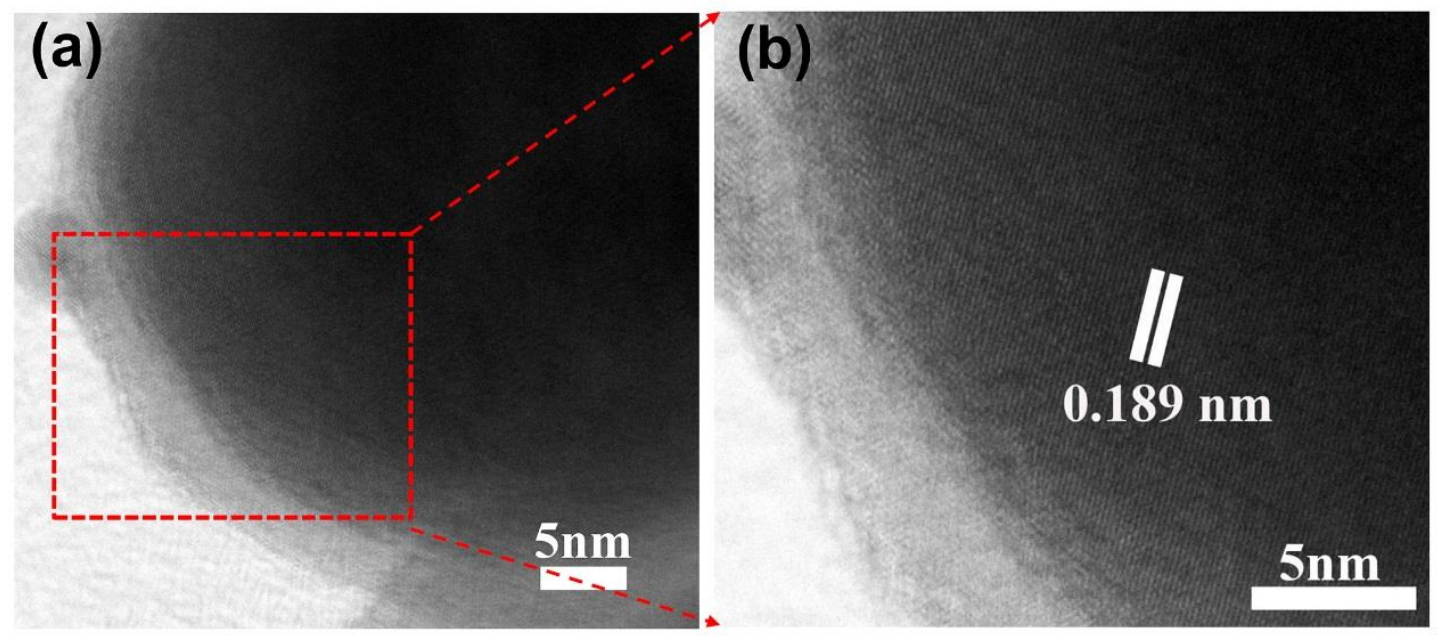

Figure S12 (a) TEM and (b) HRTEM image of the $\mathrm{Ce}_{1}$-CoP catalyst after long term test in $1 \mathrm{M} \mathrm{KOH}$ for HER. 


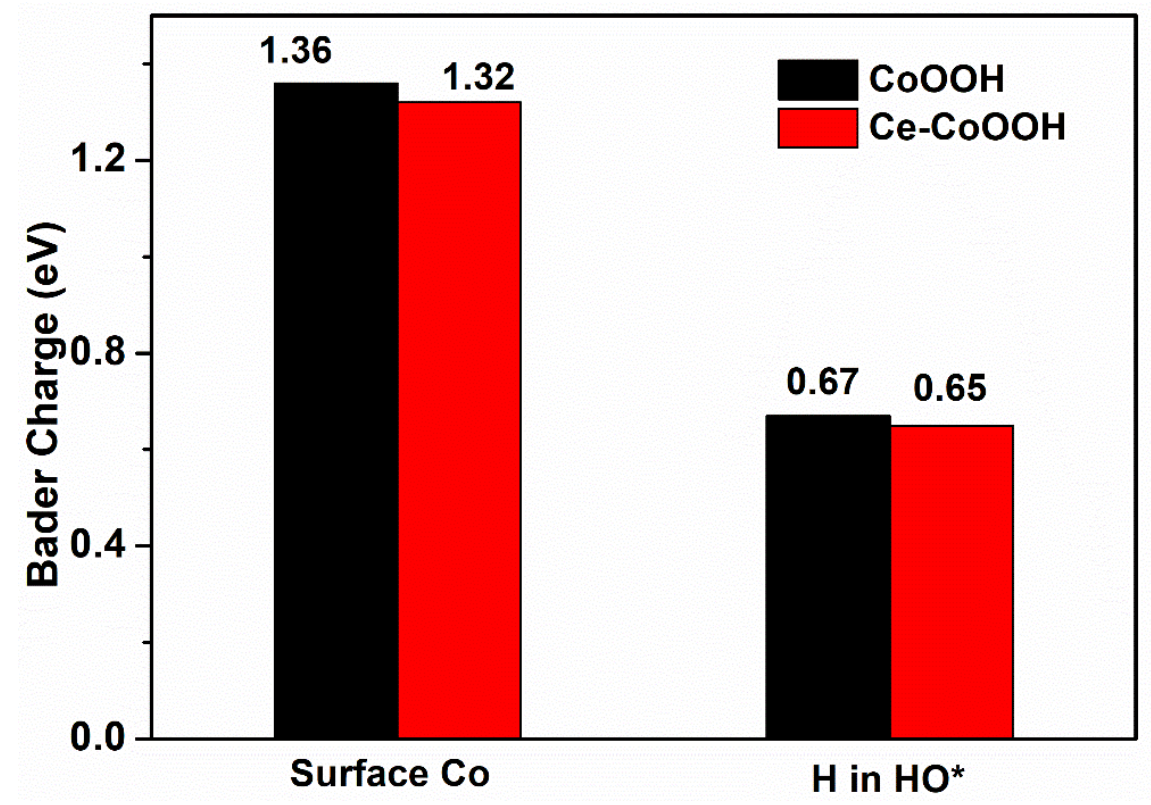

Figure S13 Bader charge analysis for surface $\mathrm{Co}$ ions and $\mathrm{H}$ in $\mathrm{HO}^{*}$ adsorption on $\mathrm{CoOOH}$ and $\mathrm{Ce}-\mathrm{CoOOH}$. 
Table S1. Summary of the elemental composition of $\mathrm{CoP}$ and $\mathrm{Ce}_{\mathrm{x}}-\mathrm{CoP}$.

\begin{tabular}{|c|c|c|c|c|c|c|}
\hline Samples & $\mathrm{C}$ & Co & $\mathbf{P}$ & $\mathbf{O}$ & $\mathbf{N}$ & $\mathrm{Ce}$ \\
\hline CoP & 11.68 & 12.24 & 29.6 & 44.91 & 1.57 & 0 \\
\hline $\mathrm{Ce}_{0.5}-\mathrm{CoP}$ & 11.58 & 12.14 & 29.33 & 44.51 & 1.46 & 0.98 \\
\hline Ce1-CoP & 12.32 & 8.71 & 30.02 & 45.76 & 1.88 & 1.31 \\
\hline $\mathrm{Ce}_{2}-\mathrm{CoP}$ & 10.29 & 11.39 & 31.0 & 43.86 & 1.73 & 1.73 \\
\hline
\end{tabular}


Table S2. Summary of various Co-based phosphides metal catalysts for HER in $1 \mathrm{M}$ $\mathrm{KOH}$.

\begin{tabular}{|c|c|c|}
\hline Catalysts & $\begin{array}{c}\text { Overpotential } \\
(\mathrm{mV}) \text { at } 10 \mathrm{~mA} \\
\mathrm{~cm}^{-2}\end{array}$ & Ref \\
\hline CoP-CC & 209 & J. Am. Chem. Soc., 2014,136, 7587. \\
\hline Nio.69Co0.31P & 167 & Nanoscale, 2016, 8, 19129. \\
\hline NiCoP/rGO & 209 & Adv. Funct. Mater., 2016, 26, 6785. \\
\hline Co-PCNFs & 249 & J. Mater. Chem. A, 2016, 4, 12818. \\
\hline $\mathrm{Co}-\mathrm{P} / \mathrm{Co}-\mathrm{PO}_{4}$ & 280 & Adv. Mater., 2015, 27, 3175. \\
\hline Co2P/Co-Foil & 157 & J. Mater. Chem. A, 2017, 5, 10561. \\
\hline $\mathrm{Cu}_{0.3} \mathrm{Co}{ }_{2.7} \mathrm{P} / \mathrm{NC}$ & 190 & $\begin{array}{c}\text { Adv. Energy Mater., 2017, 7, } \\
1601555 .\end{array}$ \\
\hline $\mathrm{Ce}_{1}-\mathrm{CoP}$ & 144 & This work \\
\hline
\end{tabular}


Table S3. Summary of various Co-based phosphides metal catalysts for OER in $1 \mathrm{M}$ $\mathrm{KOH}$.

\begin{tabular}{|c|c|c|}
\hline Catalysts & $\begin{array}{l}\text { Overpotential } \\
(\mathrm{mV}) \text { at } 10 \mathrm{~mA} \\
\mathrm{~cm}^{-2}\end{array}$ & Ref \\
\hline $\begin{array}{c}\text { MnCoP } \\
\text { nanoparticles }\end{array}$ & 330 & J. Am. Chem. Soc. 2016, 138, 4006 \\
\hline Co-P film & 340 & $\begin{array}{c}\text { Angew. Chem. Int. Ed. 2015, 54, } \\
6251\end{array}$ \\
\hline CoP/rGO hybrids & 340 & Chem. Sci. 2016, 7, 1690. \\
\hline $\begin{array}{c}\text { NiCoP } \\
\text { nanoparticle }\end{array}$ & 310 & $\begin{array}{c}\text { Adv. Mater. Interfaces 2016, 3, } \\
1500454 .\end{array}$ \\
\hline Co2P/Co foil & 319 & J. Mater. Chem. A, 2017, 5, 10561. \\
\hline Co-P/NC & 319 & Chem. Mater., 2015, 27, 7636. \\
\hline $\begin{array}{c}\text { NiCoP } \\
\text { microspheres }\end{array}$ & 340 & $\begin{array}{c}\text { Adv. Mater. Interfaces, 2016, 3, } \\
1500454 .\end{array}$ \\
\hline Ce1-CoP & 270 & This work \\
\hline
\end{tabular}


Table S4. Summary of recently reported catalysts and our sample for overall water splitting in $1 \mathrm{M} \mathrm{KOH}$.

\begin{tabular}{ccc}
\hline Catalysts & Cell voltages $(\mathbf{V})$ & Ref \\
& at $\mathbf{1 0 ~} \mathbf{~ m A ~} \mathbf{~ c m}^{-2}$ & \\
\hline CoxPO4/CoP & 1.91 & Adv. Mater. 2015, 27, 3175. \\
\hline Co-P films & 1.65 & Angew. Chem. Int. Ed.2015, 54, 6251 \\
\hline CoP/rGO & 1.7 & Chem. Sci., 2016, 7, 1690. \\
\hline CoP/GO-400 & 1.7 & Adv. Mater. Interfaces, 2016, 3, 1500454. \\
\hline CP/CNTs/Co-S & 1.743 & ACS Nano, 2016, 10, 2342. \\
\hline Iron doped CoP & 1.46 & J. Mater. Chem. A 2019, 7, 20658 \\
\hline CoP Nanoframes & 1.65 & ACS Catalysis, 2020, 10, 412 \\
\hline CoP Nanosheets & 1.6 & Adv. Funct. Mater. 2020, 30 1905252 \\
\hline Ce1-CoP & 1.65 & This work \\
\hline
\end{tabular}

